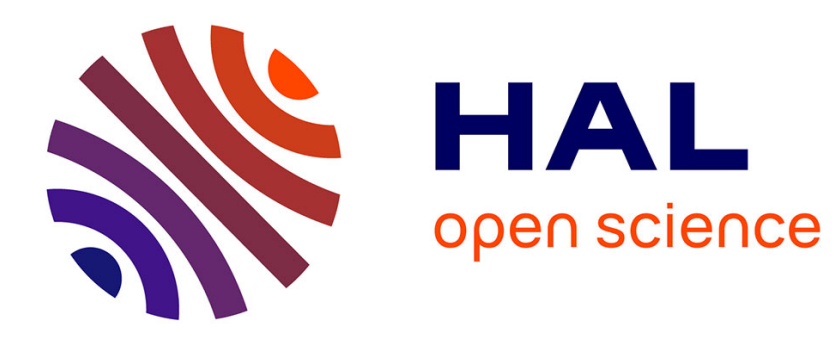

\title{
The structural language of crystalline polymers
}

Bernard Lotz

\section{To cite this version:}

Bernard Lotz. The structural language of crystalline polymers. Polymer Engineering and Science, In press, 10.1002/pen.25718. hal-03508236

\section{HAL Id: hal-03508236 \\ https://hal.science/hal-03508236}

Submitted on 3 Jan 2022

HAL is a multi-disciplinary open access archive for the deposit and dissemination of scientific research documents, whether they are published or not. The documents may come from teaching and research institutions in France or abroad, or from public or private research centers.
L'archive ouverte pluridisciplinaire HAL, est destinée au dépôt et à la diffusion de documents scientifiques de niveau recherche, publiés ou non, émanant des établissements d'enseignement et de recherche français ou étrangers, des laboratoires publics ou privés. 


\title{
The structural language of crystalline polymers.
}

\author{
Bernard Lotz \\ Institut Charles Sadron, CNRS and Université de Strasbourg \\ 23, Rue du Lœss, 67034 Strasbourg (France)
}

bernard.lotz@ics-cnrs.unistra.fr

ORCID : 0000-0001-8091-9014

RECEIVED DATE:

ABSTRACT. In sharp contrast with their amorphous counterparts, crystalline polymers provide a wealth of information on their chain conformation, the packing of the chains in unit-cells, the existence or not of mirror symmetric conformations, on the crystalline lamellae, on the folds linking the crystalline stems, etc. Three illustrations of these insights are presented here: (a) the orientation of polymer folds in crystalline lamellae, (b) the complete structural analysis of the alpha phase of isotactic polypropylene by a simple observation of the lamellar morphology and (c) the so-called lateral spread following secondary nucleation on the growth faces of a polymer crystal.

\section{Introduction.}

This special issue honoring the contributions of Greg McKenna to the understanding of polymer glasses and rheology gives me the opportunity to evoke the lasting friendly relationship we established during his stay in Strasbourg, at the laboratory of André Kovacs. André Kovacs was my $\mathrm{PhD}$ advisor and I returned to his laboratory after a two years post-doctoral stay with Doug Keith and Frank Padden at Bell Labs in Murray Hill, NJ. As one of Kovacs students, I had of course to deal with amorphous polymers and the glass transition. It was however only marginal. In my Master's thesis, I investigated by dilatometry the transitions of block-copolymers of 
polystyrene and polyethylene oxide (PS-PEO): glass transition of the PS sequences and crystallization/melting of the PEO ones. ${ }^{1}$ The $\mathrm{PhD}$ thesis was more centered on single crystals of these systems investigated by electron microscopy and electron diffraction: the crystalline part of the block copolymers had taken precedence. ${ }^{2,3}$ These same techniques were used in my post-doc to investigate the crystal structure of silks and fibrous proteins. ${ }^{4}$ They have been my favorite tools ever since.

Having had the opportunity to test and compare the level of information provided by crystalline and amorphous materials, it became clear that crystalline polymers "speak" to us - or at least to me - much more loudly than amorphous polymers. Their language needs of course to be translated, but the translation can reach an unusual level of insight - up to a detailed spelling of the words. This feast is hardly accessible to amorphous polymers since they have definitely a more limited language - which calls for a more elaborate design of experiments and a deeper evaluation of the data provided by these experiments.

In this contribution, three examples taken from our earlier work will illustrate the insights provided by crystalline polymers. They deal with (a) the orientation/structure of the folds in crystalline lamellae (b) the detailed analysis of the crystal structure of a lamellar branching observed for isotactic polypropylene in its most common alpha phase ( $\alpha \mathrm{iPP})$. This analysis helps determine the helical hand ( $\alpha$ iPP is made of right and of left-handed helices) and the orientation of each and every helix in these structures by a simple visual observation (c) the determination of the so-called "lateral spread" linked with the density of individual nucleation events on the growth faces of a crystal - a challenging issue in polymer crystallization.

\section{The structure and orientation of folds in lamellar crystals.}

When crystallized from the bulk, crystalline polymers are made of thin lamellae organized in more complex three-dimensional spherulites. Similar lamellae are produced by crystallization from solution. In the lamellae, the crystalline stems are usually normal or nearly normal to the lamellar surface. The lamellar thickness is much less than the length of the polymer chains. As a result, the chains must fold in an accordion-type manner. This conclusion was first reached by Keith Storks back in 1938 when analyzing thin films of gutta-percha. ${ }^{5}$ It was later developed by Keller (1957) for single crystals of polyethylene and for spherulites. ${ }^{6,7}$ Chain folding in polymers is ubiquitous in polymer crystallization: the surface of polymer lamellae is covered 
with an amorphous layer of less organized, amorphous material made of segments of the chain that leave and reenter the crystalline core.

For many years, the exact nature and structure of these layers remained a matter of debate - for good reasons. ${ }^{8}$ We are dealing with a thin layer, one or two molecular cross-sections thick that can hardly be investigated by experimental techniques designed to investigate bulk materials. Flory in particular advocated a highly disordered layer with "uncontrolled" orientations of folds as in the old-fashioned telephone switchboards. Crystallography-oriented colleagues considered more "organized" surfaces - if only to accommodate the density difference between the crystal core and the amorphous surface layer. ${ }^{8}$ This view had taken strength already when we introduced with Jean-Claude Wittmann the following elegant means to illustrate the folds orientation. $^{9}$

The structure of surfaces, including of thin layers, can be investigated by resorting to decoration techniques. The principle is simple. In polymer crystals, folds cannot be resolved individually. If some material is deposited (actually vaporized) on the fold surface and if it interacts with the folds, it may generate a pattern that tells something about those folds - for example their orientation. This pattern must be at a larger scale to become visible, but it can reveal much smaller and hard to identify substrate features - after all, the deposit/substrate interactions involve mostly van der Waals forces (6-8 ̊ range). The decoration technique was initially developed by Alan Bassett. ${ }^{10}$ Gold vaporized on cleaved sodium chloride single crystals moves on the surface and gathers in droplets that are aligned along cleavage steps. It was the first time a $3.5 \AA$ sized feature (the height of the cleavage steps) could be, if not "seen", at least located.

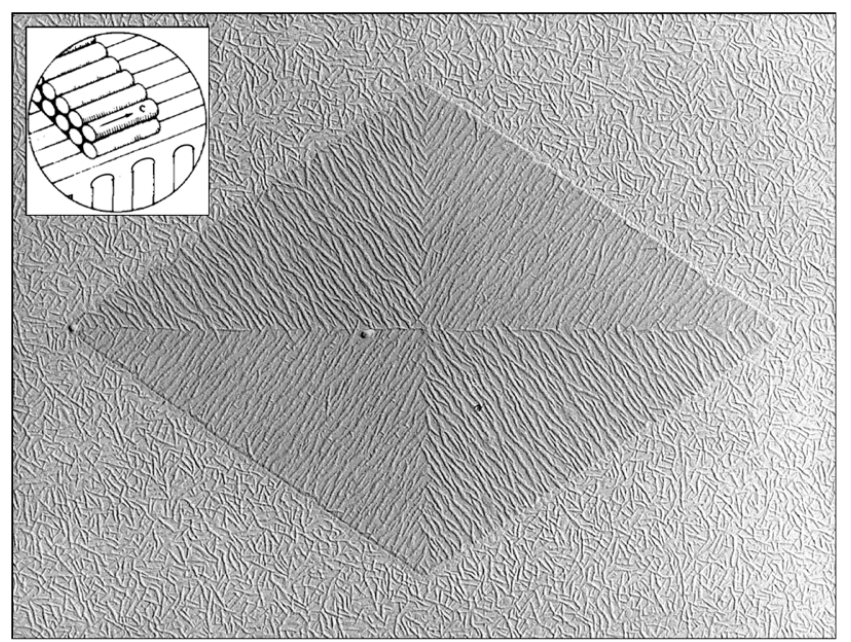

Figure 1. A solution grown single crystal of polyethylene (lateral size: $5-6 \quad m$, thickness $\approx 12$ $n m)$ decorated with polyethylene vapors. The decoration orientation indicates the orientation 
of the folds on the surface of the crystal, as (very) schematically illustrated in the inset (only the outer row of folds is shown, and furthermore in a highly idealized sharp fold version).

Applying the same technique but vaporizing polyethylene on single crystals of polyethylene (and other crystalline polymers, e.g. polyoxymethylene) yields the result shown in Figure 1. PE vapors condense and crystallize on the cold (room temperature) substrate. Crystals formed by the condensation of vapors on the glass surface are not oriented. Crystals formed on the PE fold surface are highly oriented by the substrate folds. The depositing small PE fragments end up lying flat on the surface and become oriented parallel to the folds ( $c f$. inset of Figure 1). Lateral accretion of these oriented fragments generates what is in essence piles of wood trunks - in this case lamellae edge-on. The orientation of these lamellae is at right angle to the orientation of the folds. The evidence is compelling: the folds have different orientations in the different growth sectors. They are oriented parallel to the growth faces as would be expected when a random coil is deposited and "flattens" on a flat growth face. The method can be used for bulk crystallized materials, in spherulites. It reveals a similar orientation of the folds, essentially parallel to the local growth front. ${ }^{9}$

This decoration technique has therefore a resolution of about $10 \mathrm{~nm}$ (the length of the vaporized $\mathrm{PE}$ fragments). It cannot reach the very local, nanometer range, but it confirms that a significant fraction of the folds is indeed oriented in both solution and bulk crystallization, which invalidates the switchboard model.

\section{The local structure in alpha phase iPP lamellar branching.}

The crystallographic insight to be described now can be reached only in the alpha phase of isotactic polypropylene ( $\alpha \mathrm{iPP}$ ) thanks to a unique combination of crystal structure features and growth processes.

The crystal structure of $\alpha$ iPP) was determined by Natta and Corradini in 1955, soon after the first synthesis of this polymer using a catalyst developed by Ziegler. ${ }^{11}$ The chain conformation is helical, with three motifs per turn (Figure 2, top left). Since iPP is not a chiral polymer, righthanded and left-handed helices can exist. In $\alpha \mathrm{iPP}$, these helices are organized in layers: layers of right-handed helices alternate with layers of left-handed helices along the $b$ axis of the unitcell. Furthermore, in chain axis projection, the helices pack "face-to-face" and "tip-to-tip": along the $b$ axis: the structure is actually an alternation of bilayers. The "tip" faces of the bilayers have an interesting structure: they are a perfect lozenge pattern of methyl groups. 
Indeed, the cell is monoclinic: the $a$ axis is at $\approx 100^{\circ}$ from the $c$ axis and the $a$ and c axes of the unit-cell have the same length: $\approx 6.5 \AA$.

The tip-to-tip interaction of layers made of helices of opposite hand is simple: the lozenge patterns are shifted such that the "tip" methyl groups of one layer are located at the center of the lozenge of the facing layer. The chain axes remain parallel. An original situation is created when the facing layers are made of helices of the same hand. ${ }^{12,13}$ The depositing layer must rotate around the $b$ axis in order to bring its $c$ axis parallel to the substrate $a$ axis in order to recreate an interaction similar to the crystallographic one (Figure 2, bottom left). This is a case of homo-epitaxy. ${ }^{14}$ Growth of lamellae with this different helix axis orientation generates a unique, profuse lamellar branching with branches at an $80^{\circ}$ or $100^{\circ}$ angle. This lamellar branching is also an original morphological marker. The orientation of the $c$ axis in the lamellae is known: it is normal to the lamella. In addition, in the present case, the $c$ axis orientation of one set of lamellae indicates the orientation of the $a$ axis in the other set of lamellae - an information that would be accessible only by diffraction but is reached thanks to this lamellar branching.

This information turns out to be crucial. The symmetry of the unit-cell and this monoclinic structure make it possible to determine if the cell is seen from the "front" or from the "back" of the $b$ axis, i.e. from the $b$ or the $-b$ axis. From the front, the $a$ axis appears to be oriented at 4 o'clock to the $c$ axis; from the back, it appears to be oriented at 8 o'clock to the $c$ axis (imagine a transparent clock with the long arm (the chain axis) at noon and the short arm at 4 o'clock looked at from the front or from the rear). In turn (case 1 in Figure 2) when seen from the front (or more exactly from the $+b$ side), the layers of helices with a single methyl group oriented towards the observer is made of left-handed helices, the layer showing the "back" of the helices with two methyl groups are made of right-handed helices. When (case 2 in Figure 2) the cell is seen from the $-b$ side, the helix senses are reversed: the right-handed helices have their single methyl facing the observer. 

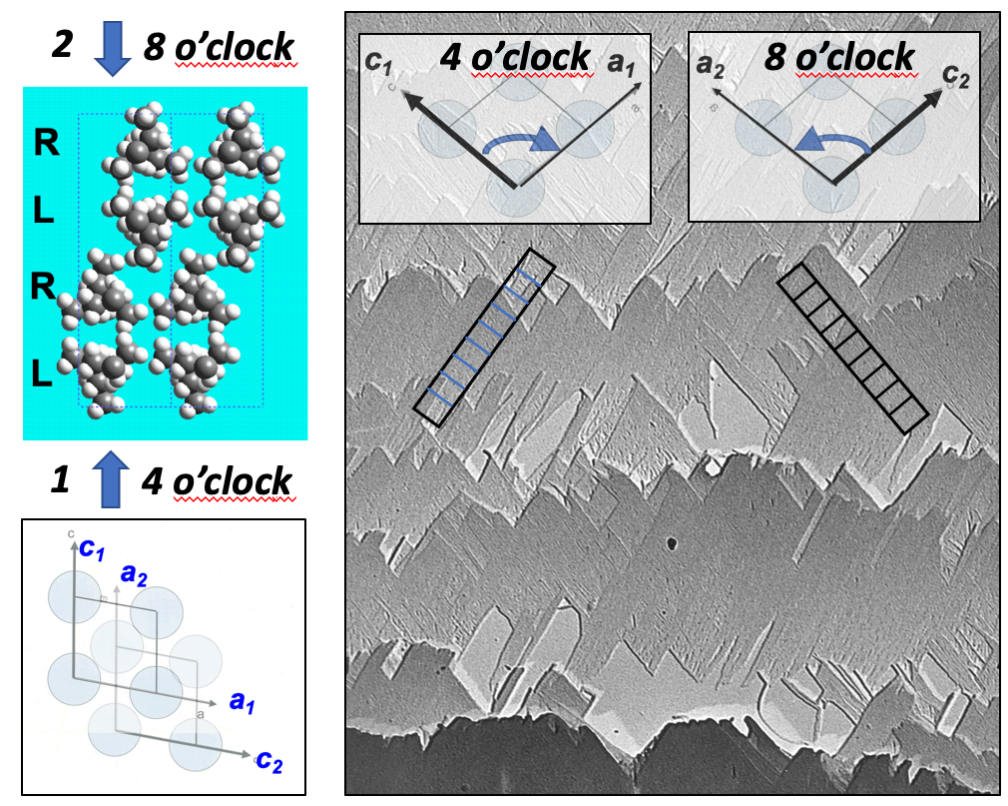

Figure 2. The crystal structure of isotactic polypropylene, alpha phase (top left) and the origin of the lamellar branching: interaction of facing layers made of helices of the same hand (bottom left). The resulting lamellar branching (right) helps determine the two unit-cell a axis orientations, parallel to the $c$ axis of the other set of lamellae. (at 4 o'clock to the $c$ axis orientation for case 1, at 8 o'clock for case 2) and the viewpoint: 1 or 2 (arrows, top left).

Figure 2 illustrates these analyses as applied to a thin film of $\alpha$ iPP crystallized on benzoic acid. iPP crystallizes epitaxially on this nucleating agent, with the $a c$ face as the contact plane - the exact orientation needed to illustrate the above features. ${ }^{14}$ The film is made of two sets of lamellae with different orientations, as also observed in solution or bulk crystallization of aiPP as a result of the homoepitaxy. The electron diffraction pattern indicates that the unit-cell is oriented as shown in the insets, with in each set the $c$ axes normal to the lamellas. In the set of lamellae oriented from bottom left to top right, the $a$ axis is at 8 o'clock to the $c$ (or helix) axis of the unit-cell: the single methyl group of left-handed helices faces the observer (case 2). In the set of lamellae oriented from the bottom left to the upper right, the $a$ axis is at 4 o'clock to the $c$ axis: the single methyl group of right-handed helices faces the observer, and left-handed helices show their back, with two methyl groups (case 1).

This analysis therefore helps determine the helical hand and the orientation of each and every stem in the whole film based on a simple observation of the branched lamellae morphology. It also confirms the structural origin of the homo-epitaxy leading to this unique lamellar branching in $\alpha \mathrm{iPP}$ : the interaction of the two sets of lamellae takes place between layers made of helices 
with the same chirality. Indeed, in the two sets of lamellae, the "tip" layers made of helices with the same hand are oriented in opposite directions $(+b$ and $-b)$ and with $\mathrm{c}$ axis orientations $\approx 100^{\circ}$ away in the $a$ c plane, which is the exact requisite needed to generate the homo-epitaxy when they interact (bottom left diagram in the figure).

Needless to say, this level of insight questions and challenges some of the very diverse views expressed about crystallization processes. One line of thought considers that polymer crystallization is a multistep process. In an initial step the macromolecular chains (pre)organize in smectic-type "bundles" that deposit on a loosely, still ill-defined growth front and reorganize to reach their final, crystallographic structure. ${ }^{15}$ This scheme has the merit of being "generic" but does not take in account the very detailed analyses provided by a more "crystallographic" approach. ${ }^{16}$ The $\alpha$ iPP crystals and lamellar branching indicates that the depositing stems "read" the surface topography and only helices with the correct hand are attached to the growth front. The selection process is even able to generate/select different stem orientations $100^{\circ}$ apart depending on the helical hands of the depositing chains and substrate layer. Such a major reorientation is definitely not accessible to an initial "smectic"-type structure in which the helical hand is not defined and the helices are (must be) parallel. In other words, this crystallographic analysis tends to support a step-by step (stem by stem) crystal growth process in polymers that is very similar to the classical picture of the small molecules-type crystal growth. ${ }^{16,17}$

\section{Crystallization processes: the buildup of a new layer via lateral spread/growth.}

The diffraction patterns of single crystals are made of a number of spots that correspond to the diffraction from specific planes. In so-called electron microscopy dark field imaging, it is possible to select individual spots and enlarge them to produce a picture of the crystal made only of the electrons diffracted by the selected diffracting planes. The crystal thus appears in "dark field" since the main electron beam is blocked by the selection aperture. This feature makes it possible to "see" the crystal interior and investigate local features such as defects, etc. In the case developed now, it helped reach a "hidden" feature of the crystal growth process.

Crystal growth amounts to building a wall with identical bricks. This is a layer by layer process: a first brick is used to start a new layer - the secondary nucleation process. Other bricks are lined up on both sides next to it - the so-called lateral spread. The lateral spread is limited by 
the density of secondary nucleation events taking place on the new layer - by the number of workers who build the wall and start the new layer. Since only the overall crystal growth rate can be measured, there is no means to determine whether a given growth rate results from limited nucleation and large spread or from dense nucleation and limited spread: there is $a$ priori no means to access such fundamental variables in crystal growth since, once deposited on the growth face, the individual constituents - the bricks or crystalline stems - are all alike.

One means to measure lateral spread does exist: it is to give to only one of the workers bricks of a different color at some stage during the building process. In crystallography, such a difference may be observed in growth twins - i.e. a sudden change in the lattice orientation. For the present purpose however, twinning must be a mild perturbance and affect the growth process in a minimal way. Such a system was found with a crystal structure of isotactic poly(vinylcyclohexane) (iPVCH) (PVCH is polystyrene with fully hydrogenated benzene rings). ${ }^{18}$ The crystal structure of interest is tetragonal, the chain conformation has a four-fold symmetry and (but!) in $c$ axis projection the chains are not parallel to the $a$ and $b$ axes (cf. Figure 3, top left). The two slight rotations of the stems (clock and anti-clock) in an otherwise identical unit-cell are the two different "colors" needed to identify them by dark field imaging.
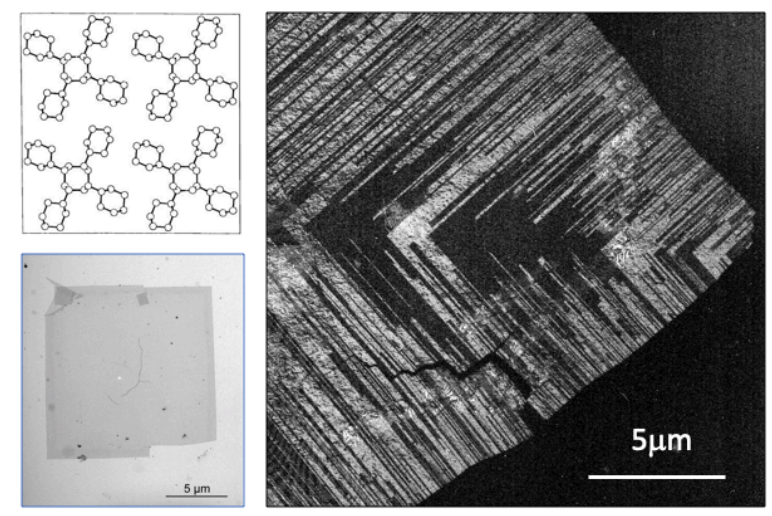

Figure 3. The crystal structure of isotactic poly(vinylcyclohexane) (iPVCH) (top left). Note the rotation (here anticlockwise) of the helices relative to the tetragonal unit cell edges. Clockwise rotation corresponds to the twinned structure. A single crystal of isotactic poly(vinylcyclohexane) as seen by electron microscopy in bright field (lower left)) and dark field through a 240 reflection. This reflection is sensitive to the azimuthal setting of helices in the unit-cell and is therefore well suited to reveal twinned domains. Note that such domains appear randomly during crystal growth (their relative distance from the crystal center is variable) but once formed, they continue to grow as twinned domains. This crystal was produced in a squalene solution at $180^{\circ} \mathrm{C}$. 
As shown in Figure 3 (bottom left), the solution grown single crystals are square and virtually featureless when examined in conventional transmission electron microscopy. When examined in dark field however (by selecting a diffraction spot that differentiates the stems "color") the crystal is clearly made of multiple narrow domains oriented normal to the growth fronts. ${ }^{19}$ The breadth corresponds to the contributions of individual twinned secondary nucleation events and their associated lateral spreads (the twinned orientation is maintained during further growth since the incoming stems reproduce the substrate stems orientation, which explains that the domains remain twinned in later growth). Similar pictures can be taken for different Tcs. Quite expectedly, the secondary nucleation events increase with decreasing crystallization temperature: the average breadth of the twinned domains decreases, from $\approx 70$ stems at $200^{\circ} \mathrm{C}$ to $\approx 30$ stems at $120^{\circ} \mathrm{C}$.

It should be emphasized that such insights cannot be reached for many (or more exactly, up to the present time, for any) other crystalline polymer. Very frequently, twinning induces more important crystallographic differences/disturbances that perturb the growth process. The iPVCH polymer, and only in this particular crystal structure, has the right combination of cell symmetry and limited disturbance to help reach this otherwise inaccessible feature of the crystallization process.

\section{Conclusion.}

The present contribution is of course far removed from the major interests of the community of colleagues working on polymer processing (only structures "at rest" are considered) or on glasses and rheology - the major themes of this special issue. It has attempted to show, in simple words, that crystalline polymers are indeed complex, but at the same time very friendly materials. They let us reach their intimacy at levels that are hardly accessible to other systems. Their structure at different length scales, from the unit-cell to the morphology (a field not covered in the present account) "speaks" to us - provided of course that we are able to decipher their language. Most useful in this respect is the use of electron microscopy and diffraction, techniques well adapted to the length scales of polymer morphologies, and even more so to polymer single crystals. The variety of chemical and crystal structures, of cell geometries, etc. is also a considerable advantage. It allows selecting the proper polymer to investigate specific problems such as the impact of folds on the lamellar shape (twisted, scrolled, etc.) ${ }^{20,21}$ or, as illustrated here, the extent of lateral spread during crystallization. In short, as indicated in the 
title of this contribution, crystalline polymers are very talkative about their structure, crystallization behavior, etc.

By contrast, amorphous polymers are, to me at least, almost mute. This is why this short contribution cannot end without conveying my sincere admiration for the work performed on polymer glasses and rheology. The challenges are considerable because the information provided by these systems remains very global, which makes it difficult to reach the intimate knowledge we all aim for. As illustrated by the contributions of André Kovacs, Greg and many others, clever instrumental designs and experimental protocols had to be, and have been, developed to understand, also, their language.

\section{References.}

(1) Lotz, B.; Kovacs, A.J.; Phase transitions in block-copolymers of polystyrene and polyethylene oxide, Polym. Prep. Am. Chem. Soc. Div. Polym. Chem., 1969, 10, 820-825

(2) Properties of copolymers composed of one poly-ethylene-oxide and one polystyrene block. II. Morphology of single crystals, Lotz, B.; Kovacs, A.J.; Basset, G.A.; Keller, A. Kolloid-Z.u.z. Polym., 1966, 209, 115-128

(3) Kovacs, A.J.; Lotz, B.; Keller, A. Multiple twinning in polyethylene oxide single crystals. A scheme for the formation of growth twins from self-seeding nuclei, J. Macromol. Sci.-Phys., 1969, B3, 385-425

(4) Lotz, B.; Keith, H.D. Crystal structure of poly(L-ala-gly) II. A model for silk I, J. Mol. Biol., 1971, 61, 201-215

(5) Storks, K. H. An Electron Diffraction Examination of Some Linear Polymers JACS, 1938, 1753

(6) Keller, A. A note on single crystals in polymers: Evidence of a folded-chain configuration. Philos. Mag. 1957, 2, 1171-1175

(7) Keller, A. Polymer crystals. Rep. Prog. Phys. 1968, 31, 623-704.

(8) Organization of macromolecules in the condensed phase, Disc. Faraday Soc., 1979, 68.

(9) Wittmann, J.C.; Lotz, B. Polymer decoration: the orientation of polymer folds as revealed by the crystallization of polymer vapors, J. Polym. Sci., Polym. Phys. Ed., 1985, 23, $205-226$ 
(10) Bassett, G. A. A new technique for decoration of cleavage and slip steps on ionic crystal surfaces Philos. Mag.1958, 3 (33), 1042-1045

(11) Natta, G.; Corradini, P. Nuovo Cim. Suppl., 1960, 15,40\$\$

(12) Wittmann, J.C.; Lotz, B. The molecular origin of lamellar branching in the $\alpha$ (monoclinic) form of isotactic polypropylene, J. Polym. Sci., Part B: Polym. Phys., 1986, 24, $1541-1558$

(13) Lotz, B.; Wittmann, J.C.; Lovinger, A.J. Structure and morphology of poly(propylenes): a molecular analysis, Polymer, 1996, 37, 4979-4992

(14) Wittmann, J.C.; Lotz, B. Epitaxial crystallization of polymers on organic and polymeric substrates, Prog. Polym. Sci., 1990, 15, 909-948

(15) Strobl, G. Crystallization and melting of bulk polymers: New observations, conclusions and a thermodynamic scheme Prog. Polym. Sci. 2006, 31, 398-442.

(16) Lotz, B. What can polymer crystal structure tell about polymer crystallization processes? Eur. Phys. J. E: Soft Matter, 2000, 3, 185-194

(17) Lotz, B.; Cheng, S.Z.D. Polymer crystallization processes as seen from the growth front's perspective, Polymer J., 2008, 40 (9) 891-899

(18) Alcazar, D.; Ruan, Jr-J.; Thierry, A.; Kawaguchi, A.; Lotz, B. Polysynthetic twinning in poly(vinylcyclohexane) single crystals and "fractional" secondary nucleation in polymer crystal growth, Macromolecules, 2006, 39, 1008-1019

(19) Alcazar, D.; Thierry, A.; Schultz, P.; Kawaguchi, A.; Cheng, S.Z.D.; Lotz, B. Determination of the extent of lateral spread and secondary nucleation density in polymer single crystal growth, Macromolecules, 2006, 39, 9120-9131

(20) Lotz, B.; Cheng, S.Z.D. A critical assessment of unbalanced surface stresses as the mechanical origin of twisting and scrolling of polymer crystals, Polymer, 2005, 46, 577-610

(21) Lotz, B.; Cheng, S.Z.D.; Li, C.Y. Structure of Negative Spherulites of Even-Even polyamides. Introducing a Complex Multicomponent Spherulite Architecture Macromolecules, 2018, 51, 5138-5156 